\title{
Alignment of the CMS muon detectors
}

\author{
Jordi Duarte Campderros ${ }^{1}$ (for the CMS Collaboration) \\ Instituto de Física de Cantabria \\ Avenida Los Castros s/n, Santander, Spain \\ E-mail: duartedifca.unican.es
}

\begin{abstract}
The CMS Muon system consists of 250 drift tube (DT) chambers in the central region and 468 cathode strip chambers (CSC) in the forward region, complimented by 480 fast-response resistive plate chambers distributed in both regions for triggering purposes. The muon system provides fast muon trigger, muon identification, and muon trajectory measurements. The performance of the muon system depends on the precise knowledge of the positions and orientations of all its elements within the CMS detector. We present two alignment techniques, track-based and hardware-based. The track-based technique uses muon tracks from pp collision data at the LHC to align the muon system elements relative to the CMS inner silicon tracker. A complimentary hardware-based technique consists of two separate optical systems in the central and forward regions linked by a third system to the inner tracker. The hardware systems are designed to perform well in an environment of large radiation flux and high magnetic field. We discuss the alignment results after several years of CMS operation, the achieved precision of the alignment techniques and the resulting impact on muon momentum resolution.
\end{abstract}

The European Physical Society Conference on High Energy Physics

18-24 July, 2013

Stockholm, Sweden

\section{$1 \quad$ Speaker}




\section{Introduction}

In the CMS detector, the muon chamber positions can be several $\mathrm{mm}$ away from their design positions due to several reasons: large deformations induced by the huge gravitational and magnetic forces acting on the detector, repositioning after detector is open and closed, imperfect positioning during installation, or thermal effects. Precise measurement of muons up to the $\mathrm{TeV}$ momentum range requires the positions and orientations of muon chambers to be known with an accuracy of a few hundred microns, comparable to their intrinsic spatial resolution. In order to achieve this, the CMS alignment strategy combines precise survey and phtogrammetry information, measurements from an optical-based hardware alignment system, and the results of alignment procedures based on muon tracks.

\section{Hardware alignment}

The hardware alignment system is composed of three separate subsystems. The endcap system aligns approximately $1 / 6$ of the CSC chambers relative to each other. Each endcap station is monitored by three radial cross-hair laser straight line monitors running along the full diameter of the supporting disks and measured by two DCOPS mounted on each of four crossed chambers. The unmeasured chambers are aligned relative to the measured ones using tracks that pass throught their overlapping regions. The system is complemented by clinometers, axial distance sensors and axial laser "transfer lines" which relate fiducial structures (transfer plates) on separate endcap stations.

The barrel system monitors the position of all 250 DT chambers using a redundant optical network. Each DT is equipped with LED light sources visible from both axial directions and observed by 600 small videocameras distributed along 36 rigid structures (MABs) mounted on the faces of the barrel wheels.

Finally, a link system simultaneously measures 12 MABs on the two external wheels and 24 CSC chambers in the first endcap muon stations and relates them to the inner silicon tracker using laser beams emitted from two rigid structures called alignment rings located at the external faces of the tracker.

A dedicated program is then used to transform the various measurements into a reconstruted DT and CSC aligned geometry. Further details about the hardware alignment system can be found in [1]. The relative precision achieved by the hardware system is about 500 $\mu \mathrm{m}$ in the azimuthal ( $\mathrm{r} \phi$ ) position, which is directly related to the curvature measurement. Although the precision is not as good as that obtained using muon tracks, the hardware system provides almost online relative displacement monitoring, is able to produce a geometry as soon as the detector is closed, and provides independent measurements which help constrain weak mode effects intrinsic of a track-based alignment.

\section{Track-based alignment}

The current track-based alignment takes the hardware-based alignment as a starting geometry and uses the inner silicon tracker as a "perfect" reference to determine the positions of all CSC and DT chambers independently. In this simplified approach, track parameters are fixed and the residuals (the difference between the extrapolated track and the track measured by each chamber) are directly related to the chamber position as shown in Figure 1. Residual distributions have non-zero widths due to multiple scattering effects and non-zero means due to 
misalignments. About ten thousand tracks (of some minimum quality) per chamber are necessary to achieve good statistical precision. The optimal chamber position is obtained by minimizing an objective function defined as

$$
\chi^{2}=\sum_{\mu}\left(\vec{R}_{\mu}-\mathbf{A}_{\mu} \boldsymbol{\delta}\right)^{T} \mathbf{E}_{\mu}\left(\vec{R}_{\mu}-\mathbf{A}_{\mu} \boldsymbol{\delta}\right)
$$

where the sum is over the number of muon tracks, $\mathbf{E}$ is the error matrix, $\mathbf{A}$ is the matrix shown in figure $1, \vec{R}$ is the residual four-vector and $\vec{\delta}$ is the chamber position six-vector. Further details about the track-based muon alignment can be found in [2].

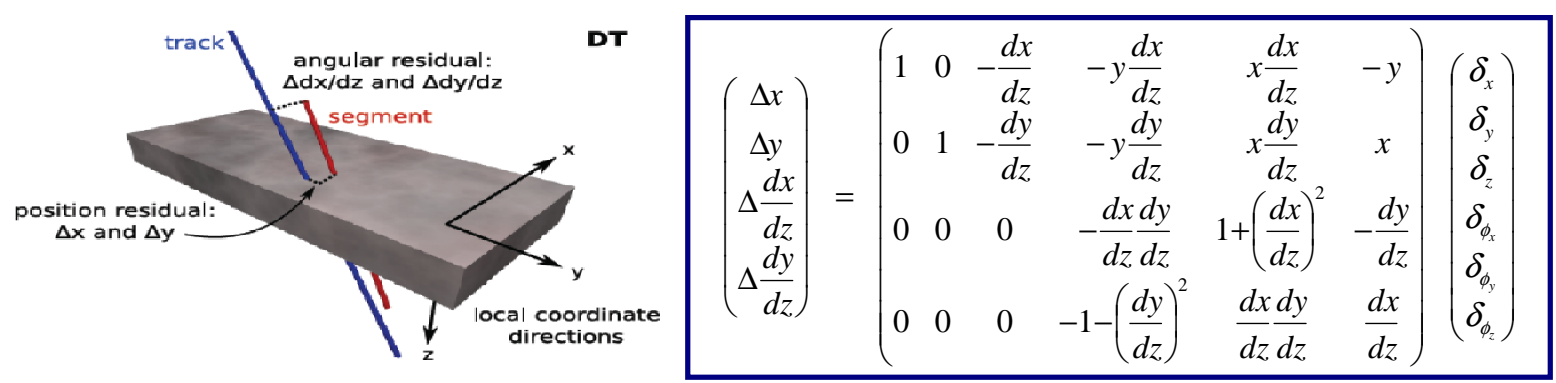

Figure 1. Illustration of the four residuals measured by a DT chamber (left) and relation between the residual four-vector and the chamber's position six-vector (right).

The precision achieved by this method is roughly 100 (150) $\mu \mathrm{m}$ in $\mathrm{r} \phi$ and $200(140) \mu \mathrm{rad}$ in $\phi_{z}$ for DT (CSC) chambers.

\section{Muon resolution}

The transverse momentum resolution using tracker-only muons and "global" muons including both tracker and muon chamber information is shown in Figure 2. This resolution is calculated by splitting cosmic ray muon tracks into separate "top" and "bottom" legs and looking at the difference in transverse momentum between them. As expected, the resolution is dominated by the tracker at low momentum, but for muons above $200 \mathrm{GeV} / \mathrm{c}$ the resolution improves when a well-aligned muon spectrometer is combined with the tracker. This is particularly crucial for high momentum muon analyses such as Z' searches [3]. 


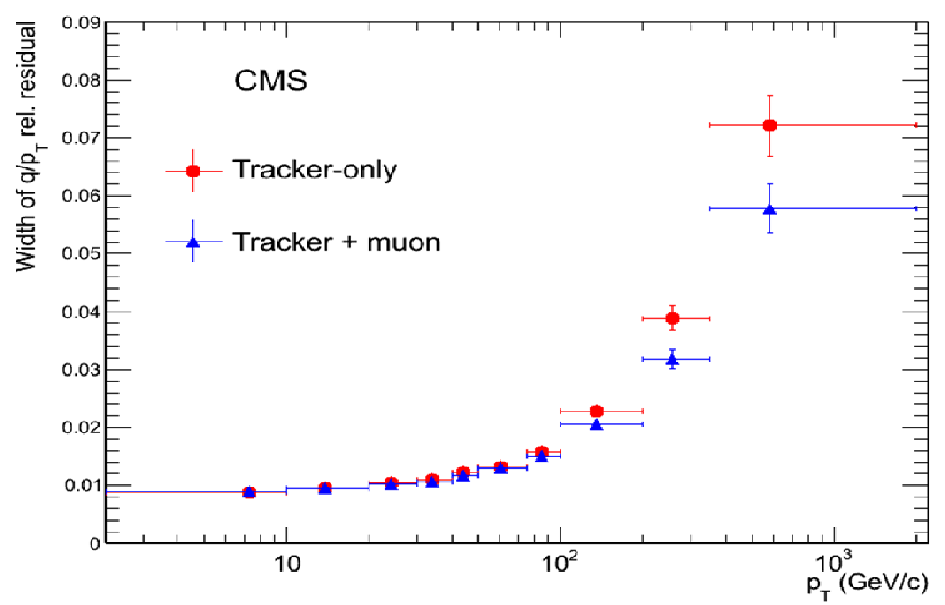

Figure 2. Transverse momentum resolution as a function of muon transverse momentum for tracker-only muons and for global muons.

\section{References}

[1] CMS Collaboration, Aligning the CMS muon chambers with the muon alignment system during an extended cosmic ray run, JINST 5 (T03019) (2010).

[2] CMS Collaboration, Alignment of the CMS muon system with cosmic-ray and beam-halo muons, JINST 5 (T03020) (2010), arXiv:0911.4022.

[3] CMS Collaboration, Search for heavy narrow dilepton resonances in pp collisions at $7 \mathrm{TeV}$ and 8 TeV centre of mass energy, Phys.Lett. B720 (2013) 63-82, doi:10.1016/j.physletb.2013.02.003, arXiv:1212.6175. 